\title{
Invariant curves for holomorphic foliations on singular surfaces
}

\author{
Edileno de Almeida Santos
}

\begin{abstract}
The Separatrix Theorem of C. Camacho and P. Sad says that there exists at least one invariant curve (separatrix) passing through the singularity of a germ of holomorphic foliation on complex surface, when the surface underlying the foliation is smooth or when it is singular and the dual graph of resolution surface singularity is a tree. Under some assumptions, we obtain existence of separatrix even when the resolution dual graph of the surface singular point is not a tree. It will be necessary to require an extra condition of the foliation, namely, absence of saddle-node in its reduction of singularities.
\end{abstract}

\section{Introduction}

In the local theory of holomorphic foliations, one of the first questions was about the existence of invariant curve (separatrix) through isolated singularity of a holomorphic vector field on $\left(\mathbb{C}^{2}, 0\right)$. This problem was posed and investigated by $\mathrm{C}$. Briot and J. Bouquet in 1854. The definitive solution was given many years later, in 1982, when C. Camacho and P. Sad showed in [4] the following

Theorem. (Separatrix Theorem) Let $v$ be a holomorphic vector field defined on neighbourhood of $0 \in \mathbb{C}^{2}$ and with isolated singularity at the origin. Then there exists an invariant curve for $v$ through $0 \in \mathbb{C}^{2}$.

In 1988, C. Camacho showed in [3] an extension of this result to include foliations on some singular surfaces:

The author would like to thanks J. V. Pereira for incentive, valuable ideas and conversations.

Key words and phrases: foliations, invariant curves, birational geometry. 2010 Mathematics Subject Classification: 37F75. 
Theorem. Let $X$ be a complex normal irreducible surface. Suppose the dual graph of resolution singularity at $p \in X$ is a tree. Then any germ of holomorphic foliation, singular at $p$, has an invariant curve through $p$.

A very elegant proof was given by M. Sebastiani in [9] (see also the exposition of M. Brunella in [2]). In the non singular case, M. Toma in [10] and J. Cano in [5] gives alternative proofs. Here we explore the surface singular case, but without the hypothesis on the dual graph.

A holomorphic foliation $\mathcal{F}$ on a smooth complex surface $X$ is given by an open covering $\left\{U_{i}\right\}$ and holomorphic vector fields with isolated singularities $v_{i}$ over each $U_{i}$ such that whenever $U_{i} \cap U_{j} \neq \varnothing$ there exists an invertible holomorphic function $g_{i j}$ satisfying $v_{i}=g_{i j} v_{j}$. The collection $\left\{g_{i j}^{-1}\right\}$ defines the holomorphic line bundle $T_{\mathcal{F}}$, called the tangent bundle of $\mathcal{F}$. The dual of $T_{\mathcal{F}}$ is the cotangente bundle $T_{\mathcal{F}}^{*}$, also called the canonical bundle $K_{\mathcal{F}}$. Recall that a reduced foliation $\mathcal{F}$ is a foliation such that every singularity $p$ is reduced in Seidenberg's sense: there is a vector field $v$ generating $\mathcal{F}$ on neighbourhood of $p$ such that the eigenvalues of the linear part of $v$ are 1 and $\lambda$, where $\lambda$ is not a positive rational number. If $\lambda=0$, the singularity $p$ is called a saddle-node; otherwise it is called a nondegenerate singularity. The well-known Seidenberg's Theorem states that after a finite sequence of blowing-ups over a singularity $p$ the induced foliation is reduced along the strict transform of $p$ (see Theorem 1, [2], p. 13). This process is called reduction of singularities.

If $X$ is a surface with only normal singularities, a holomorphic foliation on $X$ is a holomorphic foliation on $X-\operatorname{Sing}(X)$. The tangent sheaf $T_{\mathcal{F}}$ and the normal sheaf $N_{\mathcal{F}}$ are defined by taking the direct images via the inclusion $X-\operatorname{Sing}(X) \rightarrow X$ of the tangent and normal sheaves of the underlying foliation on $X-\operatorname{Sing}(X)$.

In [3], C. Camacho gives an example of non existence of separatrix. Let $\pi$ : $Y \rightarrow X$ be a resolution of the normal surface singularity $p \in X$, where $\pi^{-1}(p)=E=$ $E_{1}+E_{2}+E_{3}$ is a curve with simple normal crossings and each $E_{j}$ is a smooth rational curve, with self-intersections $E_{1}^{2}=-2, E_{2}^{2}=-2$ and $E_{3}^{2}=-3$. To find a reduced foliation without saddle-node on neighbourhood of the curve $E$ such that $E$ is $\mathcal{F}$-invariant and $\operatorname{Sing}(\mathcal{F}) \cap E=\operatorname{Sing}(E)$, consider the dual graph $\Gamma$ of $E$, which is a cycle, as illustrated bellow:

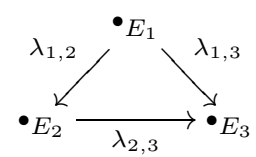

where $\lambda_{i j}=C S\left(\mathcal{F}, C_{i}, p_{i j}\right)$ is the Camacho-Sad Index (see [2]), $\left\{p_{i j}\right\}=E_{i} \cap E_{j}$. Thus $\lambda_{1,2}+\lambda_{1,3}=E_{1}^{2}, \frac{1}{\lambda_{1,2}}+\lambda_{2,3}=E_{2}^{2}, \frac{1}{\lambda_{2,3}}+\frac{1}{\lambda_{1,3}}=E_{3}^{2}$. These conditions are enough to construct the foliation on neighbourhood of the curve $E$ as desired (if the $\lambda_{i j}$ are 
not rational numbers, then the foliation will be reduced and without saddle-nod; see proof by Lins-Neto of the main theorem in [7]). In this way, if we contract the exceptional curve $E$ to the surface singular point $p$, we obtain a foliation without separatrix trough the singularity $p$.

Hence we can ask now:

What conditions can we require from the foliation to exist separatrix even when the resolution dual graph is not a tree?

First, in Section 2 we investigate relationships between foliations and their invariant compact curves by means of the logarithmic co-normal bundle, in particular we analyse the situation in which such a bundle restricted to the curve is trivial, which leads us to the notions of residues and residual representation. From these ideas, in Section 3 we explore the Separatrix Theorem. The main result is:

Theorem 1. (Theorem 3.13) Let $\mathcal{F}$ be a foliation on the normal singular surface $X$. If the foliation has no saddle-node in its resolution/reduction over the singularity $p \in X$ and the normal sheaf $N_{\mathcal{F}}$ is $\mathbb{Q}$-Gorenstein, then $\mathcal{F}$ has a separatrix through $p$.

In the above theorem, a sheaf $\mathcal{S}$ on a singular surface is called $\mathbb{Q}$-Gorenstein if there is a positive integer $k>0$ such that the $k^{t h}$ tensor power $\mathcal{S}^{\otimes k}$ is locally trivial (in particular, if $\mathcal{S}$ is trivial, then it is $\mathbb{Q}$-Gorenstein).

In this paper we assume the reader is familiar with the theory of singular holomorphic foliations on surfaces like presented by the first chapters of [2].

\section{Residues and representation}

\subsection{Basic conceptions}

Let $X$ be a smooth complex surface and let $C \subset X$ be a curve.

Definition 2.1. A logarithmic 1-form $\omega$ on an open set $U \subset X$ with poles on $C$ is a meromorphic 1-form on $U$ with the following property: for any $p \in U$ there is a neighborhood $V \subset U$ of $p$ such that

$$
\left.\omega\right|_{V}=\omega_{0}+\sum_{i=1}^{n} g_{i} \frac{d f_{i}}{f_{i}}
$$

where $\omega_{0}$ is a holomorphic 1-form on $V, f_{i}$ and $g_{i}$ are holomorphic functions on $V$ and each $f_{i}$ is a reduced equation of an irreducible component of $C \cap V$. 
Generically we are interesting in the situation where $C=\sum_{i=1}^{n} C_{i} \subset X$ is a simple normal crossing divisor, in which case we have the following exact sequence:

$$
0 \longrightarrow \Omega_{X}^{1} \longrightarrow \Omega_{X}^{1}(\log C) \longrightarrow \bigoplus_{i=1}^{n} \mathcal{O}_{C_{i}} \longrightarrow 0
$$

where $\Omega_{X}^{1}(\log C$ ) is the locally free sheaf (of rank 2) of logarithmic 1-forms (see Lemma 8.16 of [12]) and the last morphism is given by the residue, defined as follow: if locally $\left.\omega\right|_{V}=\omega_{0}+\sum_{i=1}^{n} g_{i} \frac{d f_{i}}{f_{i}}$, then the residue of $\omega$ along $C_{i}$ is given by $\left.\operatorname{Res}(\omega)\right|_{C_{i}}=\left.g_{i}\right|_{\left\{f_{i}=0\right\}}$ (see [2], pp. 78 to 83). We can see easily that this definition is independent of our choices $\left(f_{i}, g_{i}, \omega_{0}\right.$ etc. $)$.

In foliation theory we have the following definition.

Definition 2.2. Let $\mathcal{F}$ be a foliation on $X$ and suppose that the curve $C$ is $\mathcal{F}$-invariant. We say that the foliation $\mathcal{F}$ is logarithmic on $(X, C)$ when given any $p \in C, \omega$ a 1-form defining $\mathcal{F}$ on neighbourhood $V$ of $p$ and $f$ a reduced equation of $C \cap V$, the meromorphic 1-form $\frac{\omega}{f}$ is logarithmic with poles on $C$.

If the curve $C$ has only normal crossing singularities, then any reduced foliation $\mathcal{F}$ on $X$ tangent to $C$ is logarithmic on $(X, C)$. In that case, the bundle

$$
L=N_{\mathcal{F}}^{*} \otimes \mathcal{O}_{X}(C)
$$

is called logarithmic conormal bundle along $C$. If the curve is a divisor with simple normal crossing singularities, then the morphism of residue induces an exact sequence:

$$
0 \longrightarrow N_{\mathcal{F}}^{*} \longrightarrow N_{\mathcal{F}}^{*} \otimes \mathcal{O}_{X}(C) \longrightarrow \bigoplus_{i=1}^{n} \mathcal{O}_{C_{i}}
$$

Observe that a collection of local sections $\eta_{j}$ of $N_{\mathcal{F}}^{*} \otimes \mathcal{O}_{X}(C)$ defined on open sets $V_{j}$ determines a collection of residues $\operatorname{Res}_{V_{j} \cap C_{i}}\left(\eta_{j}\right)$ as local sections of $\left.N_{\mathcal{F}}^{*} \otimes \mathcal{O}_{X}(C)\right|_{C_{i}}$, $i=1, \ldots, n$.

The following normal form enable us to calculate the quotient of residues for a reduced non degenerate singularity:

Theorem 2.3. (THÉORÈME 1, [8], p. 521) Let $\mathcal{F}$ be a foliation given by a holomorphic 1 -form $\omega$ on a neighbourhood of $0 \in \mathbb{C}^{2}$ with isolated singularity at 0 and linear part $\lambda_{1} x d y-\lambda_{2} y d x$. Suppose that $\lambda_{1}, \lambda_{2}$ are not zero and $\lambda_{1} / \lambda_{2}$ and $\lambda_{2} / \lambda_{1}$ are not integers $>1$. Then there is a change of coordinates in a neighbourhood of the origin such that, in the new coordinates, the foliation is given by the 1-form

$$
\lambda_{1} x(1+x y(\ldots)) d y-\lambda_{2} y(1+x y(\ldots)) d x
$$


Example 2.4. As a consequence of the above theorem, we conclude that if the foliation $\mathcal{F}$ has a reduced nondegenerate singularity at $0 \in \mathbb{C}^{2}$, then we can find a logarithmic expression for the foliation:

$$
\eta=\lambda_{1}(1+x y(\ldots)) \frac{d y}{y}-\lambda_{2}(1+x y(\ldots)) \frac{d x}{x}
$$

in such a way that $\operatorname{Res}_{\{x=0\}}(\eta)=-\lambda_{2}$ and $\operatorname{Res}_{\{y=0\}}(\eta)=\lambda_{1}$. Hence the quotient of residues is equal to minus the quotient of eigenvalues.

Note that if the curve $C$ is compact and $\omega$ is a section of $N_{\mathcal{F}}^{*} \otimes \mathcal{O}_{X}(C)$ on neighbourhood of $C$, then the residue along each irreducible component $C_{i}$ is constant.

Fix a compact curve $C \subset X$ with simple normal crossing singularities. Let $\mathcal{F}$ be a reduced foliation tangent to $C$.

Proposition 2.5. If the reduced foliation $\mathcal{F}$ is without saddle-node in $C$, then there is a neighbourhood $U$ of $C$ and logarithmic 1-forms on open subsets of $U$ defining $\left.N_{\mathcal{F}}^{*} \otimes \mathcal{O}_{X}(C)\right|_{U}$ whose residues are locally constant.

Proof. Away from the singularities $\operatorname{Sing}(\mathcal{F}) \cap C$, we can choose local sections of the logarithmic conormal bundle with any fixed residue in $\mathbb{C}^{*}$. In neighbourhoods of the singular points, local sections with constant residues are obtained via Example 2.4.

Remember the intersection formula for normal bundle (the index $Z$ below, also called $G S V$-index, seen as a divisor, is defined in [2], pp. 24 and 25):

Theorem 2.6. (Proposition 3, [2], p. 25) Let $\mathcal{F}$ be a foliation on a complex surface $X$ and let $C \subset X$ be a smooth compact $\mathcal{F}$-invariant curve. Then we have $\left.N_{\mathcal{F}}\right|_{C}=\mathcal{N}_{C} \otimes \mathcal{O}_{C}(Z(\mathcal{F}, C))$. If $D \subset X$ is any invariant connected curve (not necessarily smooth), then $N_{\mathcal{F}} \cdot D=D^{2}+Z(\mathcal{F}, D)$.

As a consequence of Theorem 2.6, we have the following proposition:

Proposition 2.7. If the reduced foliation $\mathcal{F}$ is without saddle-node in the invariant compact curve $C=\sum_{i=1}^{n} C_{i}$, where each $C_{i}$ is a smooth curve, and $\operatorname{Sing}(\mathcal{F}) \cap$ $C=\operatorname{Sing}(C)=\bigcup_{i \neq j} C_{i} \cap C_{j}$, then $\left.N_{\mathcal{F}}^{*} \otimes \mathcal{O}_{X}(C)\right|_{C_{i}}=\mathcal{O}_{C_{i}}, i=1, \ldots, n$.

Proof. The index $Z$ is zero at any point $p \in C_{i}-C \cap \operatorname{Sing}(C)$ and equal to 1 at the points in $C_{i} \cap \operatorname{Sing}(C)$. Hence

$$
\begin{aligned}
\left.N_{\mathcal{F}}\right|_{C_{i}} & =\mathcal{N}_{C_{i}} \otimes \mathcal{O}_{C_{i}}\left(Z\left(\mathcal{F}, C_{i}\right)\right) \\
& =\mathcal{N}_{C_{i}} \otimes \mathcal{O}_{C_{i}}\left(C_{i} \cap \operatorname{Sing}(C)\right) \\
& =\mathcal{N}_{C_{i}} \otimes \mathcal{O}_{C_{i}}\left(\cup_{j \neq i} C_{j} \cap C_{i}\right) \\
& =\left.\mathcal{O}_{X}(C)\right|_{C_{i}}
\end{aligned}
$$


Remark 2.8. The above Proposition is also a consequence of (the proof of) Proposition 2.5. In fact, we note that the residues have cocycle of transition equal to the cocycle of $\left.N_{\mathcal{F}}^{*} \otimes \mathcal{O}_{X}(C)\right|_{C_{i}}$. We can choose local sections defining $N_{\mathcal{F}}^{*} \otimes \mathcal{O}_{X}(C)$ along a neighbourhood of $C_{i}$ with the same fixed residue in $\mathbb{C}^{*}$ (along $C_{i}$ ), thus we obtain the triviality of $\left.N_{\mathcal{F}}^{*} \otimes \mathcal{O}_{X}(C)\right|_{C_{i}}$.

From our discussion above we also obtain

Proposition 2.9. Suppose that the reduced foliation $\mathcal{F}$ is without saddle-node at the invariant compact curve $C=\sum_{i=1}^{n} C_{i}$, where each $C_{i}$ is a smooth curve, and $\operatorname{Sing}(\mathcal{F}) \cap C=\operatorname{Sing}(C)=\bigcup_{i \neq j} C_{i} \cap C_{j}$. Then $\left.N_{\mathcal{F}}^{*} \otimes \mathcal{O}_{X}(C)\right|_{C}=\mathcal{O}_{C}$ if, and only if, there are $\delta_{1}, \ldots, \delta_{n} \in \mathbb{C}^{*}$, open sets $V_{l}$ whose union is a neighbourhood of $C$ in $X$ and sections $\eta_{l}$ of $\left.N_{\mathcal{F}}^{*} \otimes \mathcal{O}_{X}(C)\right|_{V_{l}}$ such that

$$
V_{l} \cap C_{i} \neq \varnothing \Longrightarrow \operatorname{Res}_{C_{i}}\left(\eta_{l}\right)=\delta_{i}
$$

$i=1, \ldots, n$.

Proof. Suppose that $\left.N_{\mathcal{F}}^{*} \otimes \mathcal{O}_{X}(C)\right|_{C}=\mathcal{O}_{C}$. Taking the product of suitable constants by the local sections $\eta_{l}$ given by Proposition 2.5 , we can assume that the co-cycle of transition of residues is constant equal to 1 . Hence, in each component $C_{i}$, we have

$$
V_{l} \cap C_{i} \neq \varnothing \Longrightarrow \operatorname{Res}_{C_{i}}\left(\eta_{l}\right)=\delta_{i}
$$

for constants $\delta_{i} \in \mathbb{C}^{*}, i=1, \ldots, n$.

Reciprocally, suppose that there exist local sections $\eta_{l}$ as above. Then clearly the cocycle of transition of the residues is constant equal to 1 and hence $N_{\mathcal{F}}^{*} \otimes$ $\left.\mathcal{O}_{X}(C)\right|_{C}$ is trivial.

Remark 2.10. Let $p \in C_{1} \cap C_{2}$, where $C_{1}$ and $C_{2}$ are smooth curves. If $C=$ $C_{1} \cup C_{2}$ is invariant by a reduced foliation $\mathcal{F}$ with reduced non degenerate singularity at $p$ and $\eta$ is a local section on neighbourhood of $p$ defining $N_{\mathcal{F}}^{*} \otimes \mathcal{O}_{X}(C)$, then the quotient

$$
\operatorname{Res}_{C_{1}} \eta / \operatorname{Res}_{C_{2}} \eta
$$

is independent of $\eta$ and equal to minus the quotient of eigenvalues at $p$. This is a consequence of Theorem 2.3 (see Example 2.4).

\subsection{Residual representation}

Let $\mathcal{F}$ be a holomorphic foliation on the smooth complex surface $X$. Suppose that $\mathcal{F}$ leaves invariant a curve $C=\sum_{i=1}^{n} C_{i}$ with the following properties:

1. $C_{1}, \ldots, C_{n}$ are smooth curves; 
2. every singularity of $\mathcal{F}$ in $C$ is reduced;

3. $\operatorname{Sing}(C)=\bigcup_{i \neq j} C_{i} \cap C_{j}$ are reduced nondegenerate singularities of $\mathcal{F}$.

To simplify the exposition, we assume $\# C_{i} \cap C_{j} \leq 1$ if $i \neq j$ (there is no loss of generality, because we can do this situation after some blow-ups). If $i \neq j$ and $C_{i} \cap C_{j} \neq \varnothing$, take $\left\{p_{i j}\right\}=\left\{p_{j i}\right\}=C_{i} \cap C_{j}$.

Associated to the $\mathcal{F}$-invariant divisor $C$ we have his dual graph $\Gamma$, where each vertex correspond to an irreducible component $C_{i}$ and each edge linking two vertices correspond to the intersection of them at the point $p_{i j}$. The graph is directed, with ordering given by the ordering index from the vertices, that is, the edge $p_{i j}$ linking the vertex $C_{i}$ to the vertex $C_{j}$ has the orientation from $C_{i}$ to $C_{j}$ if $i<j$. (We make an arbitrary choice of orientation, but this is innocuous.)

To each edge $p_{i j}$, with $i<j$, we associate the complex number $\delta_{i j}=$ $-C S\left(\mathcal{F}, C_{j}, p_{i j}\right) \in \mathbb{C}^{*}$. (Note that the foliation is reduced nondegenerate at $p_{i j}$, hence

$$
\delta_{i j}=-C S\left(\mathcal{F}, C_{j}, p_{i j}\right)=-\alpha_{i j} / \beta_{i j}=\operatorname{Res}_{C_{i}} / \operatorname{Res}_{C_{j}}
$$

where $\alpha_{i j}, \beta_{i j}$ are eigenvalues at $p_{i j}$ associated to a generating vector field of the foliation.)

Thus we obtain a cohomology class $\sigma=\sigma_{(\mathcal{F}, C)} \in H^{1}\left(\Gamma, \mathbb{C}^{*}\right)$ or (equivalently) a representation $\rho=\rho_{(\mathcal{F}, C)}: \pi_{1}(\Gamma) \rightarrow \mathbb{C}^{*}$, to be called residual representation.

In some cases, the representation just presented will correspond to the obstruction for triviality of the line bundle $\left.N_{\mathcal{F}}^{*} \otimes \mathcal{O}_{X}(C)\right|_{C}$. More precisely, we have

Proposition 2.11. Suppose that the reduced foliation $\mathcal{F}$ is without saddlenode in the invariant compact curve $C=\sum_{i=1}^{n} C_{i}$, where each $C_{i}$ is a smooth curve, and $\operatorname{Sing}(\mathcal{F}) \cap C=\operatorname{Sing}(C)=\bigcup_{i \neq j} C_{i} \cap C_{j}$. Then $\left.N_{\mathcal{F}}^{*} \otimes \mathcal{O}_{X}(C)\right|_{C}=\mathcal{O}_{C}$ if, and only if, the residual representation $\rho_{(\mathcal{F}, C)}$ is trivial.

Proof. If $\left.N_{\mathcal{F}}^{*} \otimes \mathcal{O}_{X}(C)\right|_{C}=\mathcal{O}_{C}$, then the above Proposition (2.9) plus the Remark (2.10) imply that the representation $\rho_{(\mathcal{F}, C)}$ is trivial.

Reciprocally, suppose the triviality of the residual representation. Then there exist $\delta_{1}, \ldots, \delta_{n} \in \mathbb{C}^{*}$ such that

$$
\delta_{i} / \delta_{j}=\delta_{i j}=\operatorname{Res}_{C_{i}} / \operatorname{Res}_{C_{j}}
$$

Thus, it is possible to choose local sections of the logarithmic conormal bundle in neighbourhoods of the singular points of $C$ with residue $\delta_{i}$ through $C_{i}, i=1, \ldots, n$ (Remark 2.10). Away from the singularities of $C$, we can choose any residue in $\mathbb{C}^{*}$, then we take local sections with residue $\delta_{i}$ along $C_{i}$. Hence, such local sections give the trivialization of the logarithmic conormal bundle on $C$ (by residues). 
Example 2.12. If the dual graph $\Gamma$ of $C$ is a tree, then the graph is contractible (that is, $\Gamma$ has the homotopy type of a point), then obviously the representation $\rho_{(\mathcal{F}, C)}$ is trivial.

Definition 2.13. A cicly of smooth rational curves (or simply a cicly) is a union of a finite number of smooth rational curves in general position (normal crossings) $C_{i}, i=1, \ldots, m, m>1$, such that: if $m=2$, then $\# C_{1} \cap C_{2}=2$; if $m>2$, then $\# C_{i} \cap$ $C_{(i+1)}=\# C_{1} \cap C_{m}=1, i=1, \ldots, m-1$, otherwise $\# C_{i} \cap C_{j}=0$.

Example 2.14. Let $\mathcal{F}$ be a foliation tangent to a cycle of smooth rational curves $C=C_{1}+C_{2}+C_{3}$, where $C_{j}^{2}=1, j=1,2,3$, and such that $\operatorname{Sing}(\mathcal{F}) \cap C=\operatorname{Sing}(C)$ are reduced nondegenerate singularities of $\mathcal{F}$. (For example, consider the foliation given by $\omega=x d y-\lambda y d x$ on $\mathbb{P}^{2}$, where $\lambda \in \mathbb{C}-\mathbb{Q}_{\geq 0}$.)

The dual graph of $C$ is

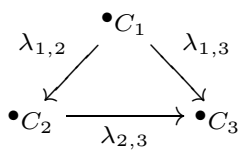

where the numbers $\lambda_{i j}$ give the correspondent cocycle in $H^{1}\left(\Gamma, \mathbb{C}^{*}\right)$ from the residual representation. Thus $\lambda_{1,2}+\lambda_{1,3}=\frac{1}{\lambda_{1,2}}+\lambda_{2,3}=\frac{1}{\lambda_{2,3}}+\frac{1}{\lambda_{1,3}}=-1$. We can see easily that in this case the representation $\rho_{(\mathcal{F}, C)}$ is always trivial. In fact, $\lambda_{1,2} \lambda_{2,3} \lambda_{3,1}=$ $\lambda_{1,2}\left(-\frac{1+\lambda_{1,2}}{\lambda_{1,2}}\right)\left(-\frac{1}{1+\lambda_{1,2}}\right)=1$.

\subsection{The Picard group of a curve}

Let $C=\sum_{i=1}^{n} C_{i}$ be a compact complex curve (connected!), where each $C_{i}$ is a smooth curve, and $\Gamma$ be the dual graph of $C$. We will describe the line bundles over $C$ with the property of triviality over each irreducible component $C_{i}, i=1, \ldots, n$.

Proposition 2.15. Let $T(C)=\left\{F \in \operatorname{Pic}(\mathrm{C}) ;\left.F\right|_{C_{i}}=\mathcal{O}_{C_{i}}, i=1, \ldots, n\right\}$. Then

$$
T(C) \simeq H^{1}\left(\Gamma, \mathbb{C}^{*}\right) \simeq \operatorname{Hom}\left(\pi_{1}(\Gamma), \mathbb{C}^{*}\right) .
$$

Proof. Consider the short exact sequence

$$
1 \longrightarrow \mathcal{O}_{C}^{*} \longrightarrow \bigoplus_{i=1}^{n} \mathcal{O}_{C_{i}}^{*} \longrightarrow \bigoplus_{p \in \operatorname{Sing}(C)} \mathbb{C}^{*} \longrightarrow 1
$$

where the morphism with image in $\bigoplus_{p \in \operatorname{Sing}(C)} \mathbb{C}^{*}$ is given by quotient (to do this we use the index order): if $k<l$ and $q \in C_{k} \cap C_{l}$, a local section of $\bigoplus_{i=1}^{n} \mathcal{O}_{C_{i}}^{*}$ with 
value $\lambda_{k}$ at $q \in C_{k}$ and $\lambda_{l}$ at $q \in C_{l}$ has image with value $\lambda_{k} / \lambda_{l}$ at $q$ (as a section of $\left.\bigoplus_{p \in \operatorname{Sing}(C)} \mathbb{C}^{*}\right)$. Hence, we have a long exact sequence in cohomology

$$
1 \longrightarrow \mathbb{C}^{*} \longrightarrow \bigoplus_{i=1}^{n} \mathbb{C}^{*} \longrightarrow \bigoplus_{p \in \operatorname{Sing}(C)} \mathbb{C}^{*} \longrightarrow \operatorname{Pic}(C) \longrightarrow \bigoplus_{i=1}^{n} \operatorname{Pic}\left(C_{i}\right) \longrightarrow 1
$$

Therefore

$$
\begin{aligned}
\operatorname{Hom}\left(\pi_{1}(\Gamma), \mathbb{C}^{*}\right) & \simeq H^{1}\left(\Gamma, \mathbb{C}^{*}\right) \\
& \simeq \frac{\bigoplus_{p \in \operatorname{Sing}(C)} \mathbb{C}^{*}}{\operatorname{Im}\left(\bigoplus_{i=1}^{n} \mathbb{C}^{*} \rightarrow \bigoplus_{p \in \operatorname{Sing}(C)} \mathbb{C}^{*}\right)} \\
& \simeq \frac{\bigoplus_{p \in \operatorname{Sing}(C)} \mathbb{C}^{*}}{\operatorname{Ker}\left(\bigoplus_{p \in \operatorname{Sing}(C)} \mathbb{C}^{*} \rightarrow \operatorname{Pic}(C)\right)} \\
& \simeq \operatorname{Im}\left(\bigoplus_{p \in \operatorname{Sing}(C)} \mathbb{C}^{*} \longrightarrow \operatorname{Pic}(C)\right) \\
& \simeq \operatorname{Ker}\left(\operatorname{Pic}(C) \longrightarrow \bigoplus_{i=1}^{n} \operatorname{Pic}\left(C_{i}\right)\right) \\
& =T(C) \square
\end{aligned}
$$

Thus, a line bundle $F \in \operatorname{Ker}\left(\operatorname{Pic}(\mathrm{C}) \rightarrow \bigoplus_{i=1}^{n} \operatorname{Pic}\left(C_{i}\right)\right)=\operatorname{Im}\left(\bigoplus_{p \in \operatorname{Sing}(C)} \mathbb{C}^{*} \rightarrow\right.$ $\operatorname{Pic}(C))=T(C)$ determines an element in $H^{1}\left(\Gamma, \mathbb{C}^{*}\right)$ (hence a representation $\rho_{F}$ : $\left.\pi_{1}(\Gamma) \rightarrow \mathbb{C}^{*}\right)$ and reciprocally an element in $H^{1}\left(\Gamma, \mathbb{C}^{*}\right)$ determines a line bundle in $T(C)$.

The interesting situation for us consists of a curve $C=\sum_{i=1}^{n} C_{i}$, with simple normal crossing singularities, invariant by a reduced foliation $\mathcal{F}$ without saddlenode and singular only at the crossing points of the curve, and the logarithmic conormal bundle $L=N_{\mathcal{F}}^{*} \otimes \mathcal{O}_{X}(C)$ in restriction to the curve $C$, which result $F=$ $\left.L\right|_{C} \in \operatorname{Ker}\left(\operatorname{Pic}(C) \rightarrow \bigoplus_{i=1}^{n} \operatorname{Pic}\left(C_{i}\right)\right)$ by Proposition 2.7 .

Finally, we can generalize the Proposition 2.11.

Proposition 2.16. Suppose that the reduced foliation $\mathcal{F}$ is without saddlenode in the invariant compact curve $C=\sum_{i=1}^{n} C_{i}$, where each $C_{i}$ is a smooth curve, and $\operatorname{Sing}(\mathcal{F}) \cap C=\operatorname{Sing}(C)=\bigcup_{i \neq j} C_{i} \cap C_{j}$. Then the line bundle $F=\left.N_{\mathcal{F}}^{*} \otimes \mathcal{O}_{X}(C)\right|_{C}$ is determined by the residual representation $\rho_{(\mathcal{F}, C)}$. That is, $\rho_{F}=\rho_{(\mathcal{F}, C)}$.

Proof. Let $B \in T(C)$. Each $B_{i}=\left.B\right|_{C_{i}}$ can be trivialized by a holomorphic morphism $\phi_{i}$ to the line bundle $C_{i} \times \mathbb{C}$. Following the isomorphism $T(C) \simeq H^{1}\left(\Gamma, \mathbb{C}^{*}\right)$ we obtain the cohomology class $\sigma_{B}$ given by $p_{i j} \rightarrow \lambda_{i j}$, where $C_{i} \cap C_{j}=\left\{p_{i j}\right\}$ and $\lambda_{i j}=\frac{\phi_{i}\left(p_{i j}, v\right)}{\phi_{j}\left(p_{i j}, v\right)}$ if $i<j$ and $v \neq 0$. If $B=F$, by Remark 2.8 we can trivialize each 
line bundle $F_{i}=\left.F\right|_{C_{i}}$ taking residues of local sections of $N_{\mathcal{F}}^{*} \otimes \mathcal{O}_{X}$, in such a way that $\lambda_{i j}=\operatorname{Res}_{C_{i}} / \operatorname{Res}_{C_{j}}=-C S\left(\mathcal{F}, C_{j}, p_{i j}\right)$. We conclude that $\sigma_{F}=\sigma_{(\mathcal{F}, C)}$ and hence $\rho_{F}=\rho_{(\mathcal{F}, C)}$.

\subsection{Residual divisor}

If the representation $\rho_{(\mathcal{F}, C)}$ is trivial, then there are $\delta_{1}, \ldots, \delta_{n} \in \mathbb{C}^{*}$ such that $\delta_{i j}=\delta_{i} / \delta_{j}$ and, in this way, we can define, unless multiplication by constant factor, the residual divisor

$$
R=\operatorname{Res}(\mathcal{F}, C)=\sum_{i=1}^{n} \delta_{i} C_{i} .
$$

In order to make precise the definition, we determine the residual divisor by the choice $\delta_{1}=1$, hence the solution is unique in $\delta_{2}, \ldots, \delta_{n}$ (obviously, this construction depends on the index order from the irreducible components of $C$ ).

Example 2.17. Suppose now

$$
D=\sum_{i=1}^{n} C_{i}+\sum_{k=1}^{m} S_{k}
$$

with $C_{1}, \ldots, C_{n}$ compact smooth curves and $S_{1}, \ldots, S_{m}$ the germs of separatrices which are not supported on $C$ trough reduced nondegenerate singularities of $\mathcal{F}$ in $C-\operatorname{Sing}(C)$. Suppose that the representation $\rho_{(\mathcal{F}, D)}$ is trivial, with residual divisor

$$
R=\operatorname{Res}(\mathcal{F}, D)=\sum_{i=1}^{n} \mu_{i} C_{i}+\sum_{k=1}^{m} \delta_{k} S_{k}
$$

Assume also that the foliation don't have weak separatrix supported on $C$. Then

$$
\begin{aligned}
R \cdot C_{j} & =\sum_{i=1}^{n} \mu_{i} C_{i} \cdot C_{j}+\sum_{j=1}^{m} \delta_{k} S_{k} \cdot C_{j} \\
& =\mu_{j}\left(C_{j}^{2}+\sum_{i \neq j} \frac{\mu_{i}}{\mu_{j}} C_{i} \cdot C_{j}+\sum_{k=1}^{m} \frac{\delta_{k}}{\mu_{j}} S_{k} \cdot C_{j}\right) \\
& =\mu_{j}\left(C_{j}^{2}-C S\left(\mathcal{F}, C_{j}\right)\right) \\
& =0
\end{aligned}
$$

for $j=1, \ldots, n$. 


\section{Existence of separatrix}

\subsection{Trivial residual representation and separatrices}

Let $\mathcal{F}$ be a holomorphic foliation on a smooth complex surface $X$. Suppose that $\mathcal{F}$ leaves invariant a compact curve $C=\sum_{i=1}^{n} C_{i}$ with the following properties:

1. $C_{1}, \ldots, C_{n}$ are smooth curves;

2. the singularities of $\mathcal{F}$ in $C$ are all reduced;

3. $\operatorname{Sing}(C)=\bigcup_{i \neq j} C_{i} \cap C_{j}$ are reduced nondegenerate singularities of $\mathcal{F}$. Write $\operatorname{Sep}(\mathcal{F}, C)=S_{1}+\ldots+S_{m}$ for the separatrices (germs) not supported on $C$ and passing trough reduced nondegenerate singularities in $\operatorname{Sing}(\mathcal{F}) \cap(C-\operatorname{Sing}(C))$.

Let $D=C+\operatorname{Sep}(\mathcal{F}, C)$ and $\rho=\rho_{(\mathcal{F}, D)}: \pi_{1}(\Gamma) \rightarrow \mathbb{C}^{*}$ be the residual representation of $\mathcal{F}$ along $D$. Obviously, $\rho_{(\mathcal{F}, D)}$ is trivial if, and only if, $\rho_{(\mathcal{F}, C)}$ is trivial.

Theorem 3.1. Suppose that $\mathcal{F}$ has no weak separatrix supported on $C$ and that the residual representation $\rho=\rho_{(\mathcal{F}, D)}$ is trivial, with residues $\mu_{1}, \ldots, \mu_{n}$ along $C$ and residues $\delta_{1}, \ldots, \delta_{m}$ along $\operatorname{Sep}(\mathcal{F}, C)$, that is, the residual divisor is

$$
R=\operatorname{Res}(\mathcal{F}, D)=\sum_{i=1}^{n} \mu_{i} C_{i}+\sum_{k=1}^{m} \delta_{k} S_{k}
$$

If the matrix of intersection $\left[C_{i} \cdot C_{j}\right]_{i j}$ of $C$ is invertible, then

$$
\# \operatorname{Sep}(\mathcal{F}, C)=m \geq \operatorname{dim}_{\mathbb{Q}}\left(\sum_{i=1}^{n} \mu_{i} \mathbb{Q}+\sum_{k=1}^{m} \delta_{k} \mathbb{Q}\right) \geq 1
$$

Proof. Consider the matrix of intersection $A=\left[C_{i} \cdot C_{j} \oplus C_{i} \cdot S_{k}\right]$ of $C$ with $D=$ $C+\operatorname{Sep}(\mathcal{F}, C)$. Take the $\mathbb{C}$-divisor in $X$ given by $R=\sum_{j=1}^{n} \mu_{j} C_{j}+\sum_{k=1}^{m} \delta_{k} S_{k}$. Hence $C_{i} \cdot R=R \cdot C_{i}=0$, for $i=1, \ldots, n$. Therefore

$$
A\left(\mu_{1}, \ldots, \mu_{n}, \delta_{1}, \ldots, \delta_{m}\right)=(0, \ldots, 0) \in \mathbb{C}^{n} .
$$

Since the matrix of intersection $\left[C_{i} \cdot C_{j}\right]$ is invertible, then the matrix $A$ has rank $n$, hence the equality above correspond to $n \mathbb{Q}$-linearly independent relations (with integer coefficients) between $\mu_{1}, \ldots, \mu_{n}, \delta_{1}, \ldots, \delta_{m}$. Then

$$
1 \leq \operatorname{dim}_{\mathbb{Q}}\left(\sum_{i=1}^{n} \mu_{i} \mathbb{Q}+\sum_{k=1}^{m} \delta_{k} \mathbb{Q}\right) \leq m=\# \operatorname{Sep}(\mathcal{F}, C) .
$$

An interesting consequence is the following

Corollary 3.2. Suppose that the representation $\rho_{(\mathcal{F}, C)}$ is trivial. If the matrix of intersection $\left[C_{i} \cdot C_{j}\right]_{i j}$ of $C$ is invertible, then there is a separatrix $S$ trough $\operatorname{Sing}(\mathcal{F}) \cap(C-\operatorname{Sing}(C))$ not supported on $C$ that is not a weak separatrix of saddlenode. 
Proof. In fact, if there is no saddle-node in $\operatorname{Sing}(\mathcal{F}) \cap(C-\operatorname{Sing}(C))$ with weak separatrix supported on $C$, then the Theorem 3.1 imply the existence of a singular point of the foliation in $C-\operatorname{Sing}(C)$ trough which pass a separatrix not supported on $C$ and which is not a weak separatrix of saddle-node. Otherwise, if there is a saddle-node in $\operatorname{Sing}(\mathcal{F}) \cap(C-\operatorname{Sing}(C))$ with weak separatrix supported on $C$, then the strong separatrix is not supported on $C$.

\subsection{The separatrix theorem}

Let $\mathcal{F}$ be a singular holomorphic foliation on the smooth complex surface $X$. Suppose that $\mathcal{F}$ leaves invariant a curve $C=\sum_{i=1}^{n} C_{i}$ with the following properties (as before):

1. $C_{1}, \ldots, C_{n}$ are smooth curves;

2. the singularities of $\mathcal{F}$ in $C$ are all reduced;

3. Sing $(C)=\bigcup_{i \neq j} C_{i} \cap C_{j}$ are reduced nondegenerate singularities of $\mathcal{F}$.

A very interesting situation is when the representation $\rho=\rho_{(\mathcal{F}, C)}$ associated to the exceptional curve is trivial. Remember this notion:

Definition 3.3. (Exceptional Curves) A compact, connected, reduced curve $C$ in a nonsingular surface $X$ is called exceptional if there is a bimeromorphism $\pi$ : $X \rightarrow Y$ such that $C$ is exceptional for $\pi$, i.e., if there is an open neighbourhood $U$ of $C$ in $X$, a point $y \in Y$, and a neighbourhood $V$ of $y$ in $Y$, such that $Y$ is normal and $\pi$ sends $U-C$ biholomorphically over $V-\{y\}$, where $\pi(C)=y$. We will also express this situation by saying that $C$ is contracted in $y$.

The following classical result is very important:

Theorem 3.4. (Grauert's criterion, [1], p. 91) A compact, connected, reduced curve $C=\sum_{i=1}^{n} C_{i}$ with irreducible components $C_{i}$ in a smooth surface is exceptional if, and only if, the matrix of intersection $\left[C_{i} \cdot C_{j}\right]$ is negative definite.

Now we suppose triviality of the residual representation and that the curve is exceptional. The theorem bellow is in fact a simple consequence of Corollary 3.2, because every negative definite matrix is invertible.

Theorem 3.5. If the curve $C$ is compact and exceptional and the representation $\rho=\rho_{(\mathcal{F}, C)}$ is trivial, then there is at last one singularity $p \in \operatorname{Sing}(\mathcal{F}) \cap(C-$ Sing $(C))$ and separatrix trough $p$ not supported on $C$ that is not a weak separatrix of saddle-node.

As a consequence we have a criterion for the existence of separatrix in singular (normal) surface. 
Corollary 3.6. Let $\mathcal{F}$ be a foliation on the singular normal surface $X$. Let $\pi: Y \rightarrow X$ be a bimeromorphism which resolve the singularity $p$ and such that the induced foliation $\mathcal{G}=\pi^{*} \mathcal{F}$ is reduced. Suppose that there exists a connected subcurve $C=\sum_{i=1}^{n} C_{i} \subset E=\pi^{-1}(p)$ such that the foliation $\mathcal{G}$ is reduced nondegenerated at the crossing points $\operatorname{Sing}(C)=\bigcup_{i \neq j} C_{i} \cap C_{j}$ and

$$
q \in \operatorname{Sing}(E) \cap(C-\operatorname{Sing}(C)) \Longrightarrow C S(\mathcal{G}, C, q)=0 .
$$

If the representation $\rho=\rho_{(\mathcal{G}, C)}$ is trivial, then $\mathcal{F}$ has a separatrix trough $p$.

Proof. The curve $C$ is exceptional, hence we can use Theorem 3.5. Thus, there exists a singularity $q \in C-\operatorname{Sing}(C)$ and separatrix (of $\mathcal{G}$ ) trough $q$ but not supported on $E$ (by hypothesis). Such a separatrix will be projected by $\pi$ to a separatrix of $\mathcal{F}$ trough $p$.

Remark 3.7. We must impose that $X$ is normal to get $E=\pi^{-1}(p)$ exceptional.

Corollary 3.8. (Generalization of the Separatrix Theorem by Camacho, [3]) Consider a complex normal irreducible surface $X$. Suppose the resolution graph of $X$ at a point $p$ is a tree, $i$. e., a finite contractible 1-dimensional complex. Then any germ of holomorphic foliation, singular at $p$, admits an invariant analytic curve through $p$.

Proof. Let $E$ be an exceptional divisor obtained by resolution of the singularity $p \in X$ and reduction of singularities of the foliation. The dual graph of $E$ is a tree, by hypothesis. It's enough to show the existence of a connected sub-curve $C \subset E$ in the conditions of the Corollary above, because such sub-curve will has also contractible dual graph, hence the representation $\rho_{(\mathcal{G}, C)}$ will be trivial.

We follow the argument of M. Toma in [10]. Let $\Gamma$ be the dual graph of $E$ and $\Lambda \subset \Gamma$ be the sub-graph obtained by the union of all edges in $\Gamma$ that do not connect vertices by a crossing in saddle-node, that is, we remove from $\Gamma$ the edges which correspond to crossing in saddle-node. Note that when we remove such edges from the connected tree we divide the tree in two connected components, one is the component of the strong separatrix and the other is the component of the weak separatrix. Making this process and always fixing the component of the strong separatrix, at the end we obtain a connected component $\Gamma_{0} \subset \Lambda$ without vertices that are weak separatrices. Then $\Gamma_{0}$ is the dual graph of a sub-curve $C$ with the desired properties.

In particular, we have the important

Corollary 3.9. (Separatrix Theorem of Camacho and Sad, [4]) Let $\mathcal{F}$ be a foliation on the smooth surface $X$ and $p \in \operatorname{Sing}(\mathcal{F})$. Then there exists a separatrix of $\mathcal{F}$ through $p$. 


\subsection{Torsion residual representation and separatrices}

We begin with the familiar covering trick in its simplest version (non-branched).

Proposition 3.10. Let $X$ be a complex manifold and let $L$ be a line bundle over $X$ of order $k>0$ in $\operatorname{Pic}(X)$, that is, $k$ is the smaller positive integer such that $L^{\otimes k}$ is trivial. Then there is a regular cyclic covering of order $k, g: Y \rightarrow X$, such that $g^{*} L$ is trivial.

Proof. See [1], p. 54 .

Remember also the following result from Complex Geometry:

Theorem 3.11. (Grauert, Satz 5, [6], p. 340) Let $A$ be a compact connected analytic subset of the analytic variety $X$. Then $A$ is an exceptional variety if and only if it has a strongly pseudoconvex neighbourhood $G$ in $X$ such that $A$ is the maximal compact analytic subset of $G$.

As a consequence, in the particular case of our interest, we obtain the following fact: if $g: Y \rightarrow X$ is a finite covering of the complex surface $X$ and $C \subset X$ is an exceptional curve, then $g^{-1}(C)$ is an exceptional curve in $Y$.

Suppose that the foliation $\mathcal{F}$ leaves invariant a curve $C=\sum_{i=1}^{n} C_{i}$ such that:

1. $C_{1}, \ldots, C_{n}$ are smooth curves;

2. all singularities of $\mathcal{F}$ in $C$ are reduced nondegenerated.

With this hypotheses, we obtain a version of Theorem 3.5 in the case of torsion residual representation.

Theorem 3.12. If the $\mathcal{F}$-invariante curve $C=\sum_{i=1}^{n} C_{i} \subset X$ is compact and exceptional and the representation $\rho=\rho_{(\mathcal{F}, C)}$ is torsion of order $k$, then there is at least one singularity $p \in \operatorname{Sing}(\mathcal{F}) \cap(C-\operatorname{Sing}(C))$ and separatrix trough $p$, not supported in $C$.

Proof. Suppose, by contradiction, that there is no singularity of the foliation in $C-\operatorname{Sing}(C)$. Let $F=F_{\rho}=\left.N_{\mathcal{F}}^{*} \otimes \mathcal{O}_{X}(C)\right|_{C}$ be the line bundle over $C$ associated to $\rho$ (see the subsection 2.3). For each $C_{i}$, consider a tubular neighbourhood $U_{i} \subset X$ of $C_{i}$. If the open subsets $U_{i}$ are small enough, the tubular projections can be glue together by means of suitable "surgery" at neighbourhoods of the singular (crossing) points, and we obtain a projection from $U=\cup_{i} U_{i}$ to $C$, that is, a deformation retract of $U$ onto $C$. Hence $U$ and $C$ has the same homotopy type, therefore $H^{1}\left(U, \mathbb{C}^{*}\right) \simeq \operatorname{Hom}\left(\pi_{1}(U), \mathbb{C}^{*}\right) \simeq \operatorname{Hom}\left(\pi_{1}(C), \mathbb{C}^{*}\right) \simeq H^{1}\left(C, \mathbb{C}^{*}\right)$, and we can consider a $\mathbb{C}^{*}$-flat extension $L$ of $F$ for $U$ in such a way that $L^{\otimes k}$ is trivial. Then, by the covering trick mentioned at the beginning, we obtain a cyclic covering of order $k$ from a surface $Y$ over $X$, say $g: Y \rightarrow X$, with $g^{*} L$ trivial. 
Consider $\mathcal{G}=g^{*} \mathcal{F}$ and $D=\sum_{j=1}^{k n} D_{j}=g^{-1}(C)$. Since we assume $\operatorname{Sing}(\mathcal{F}) \cap(C-$ $\operatorname{Sing}(C))=\varnothing$, then also $\operatorname{Sing}(\mathcal{G}) \cap(D-\operatorname{Sing}(D))=\varnothing$. Thus

$$
\left.N_{\mathcal{G}}^{*} \otimes \mathcal{O}_{Y}(D)\right|_{D}=g^{*} F=\left.g^{*} L\right|_{D}=\mathcal{O}_{D}
$$

hence the representation $\tilde{\rho}=\rho_{(\mathcal{G}, D)}$ is trivial (see the subsection 2.3 and the Proposition 2.16). By Theorem 3.11, the curve $D$ is exceptional. Therefore, from Theorem $3.5, \operatorname{Sing}(\mathcal{G}) \cap(D-\operatorname{Sing}(D)) \neq \varnothing$, a contradiction.

\subsection{Foliations with $\mathbb{Q}$-Gorenstein normal bundle}

A sheaf $\mathcal{S}$ on a singular surface is called $\mathbb{Q}$-Gorenstein if there is a positive integer $k>0$ such that the $k$-th tensor power $\mathcal{S}^{\otimes k}$ is locally trivial. In particular, if the sheaf is trivial, it is $\mathbb{Q}$-Gorenstein.

Now we develop the proof of the Theorem 1:

Theorem 3.13. (Theorem 1) Let $\mathcal{F}$ be a foliation on the normal singular surface $X$. If the foliation has no saddle-node in its resolution/reduction over the singularity $p \in X$ and the normal sheaf $N_{\mathcal{F}}$ is $\mathbb{Q}$-Gorenstein, then $\mathcal{F}$ has a separatrix through $p$.

To prove the theorem, we need some previous results.

Lemma 3.14. (Lemma 5 of [11]) Let $X$ be a smooth compact complex surface, $K$ a compact subset of $X$ and $B$ be a holomorphic vector bundle over $X$. If $X-K$ is strictly pseudoconvex, then every section $s$ of $B$ over $X-K$ can be extended as a meromorphic section $\tilde{s}$ over all $X$.

Proposition 3.15. Let $\mathcal{F}$ be a foliation on the singular normal surface $X$. Let $f: Y \rightarrow X$ be a resolution singularity at $p \in X$. If the normal sheaf $N_{\mathcal{F}}$ is $\mathbb{Q}$-Gorenstein, then, in neighbourhood $V$ of $E$,

$$
\left.N_{\mathcal{G}}^{\otimes k}\right|_{V}=\left.\mathcal{O}_{Y}\left(\sum_{i=1}^{n} a_{i} E_{i}\right)\right|_{V}
$$

where $k$ is the smallest integer for which $N_{\mathcal{F}}^{\otimes k}$ is trivial in neighbourhood of $p$, the $a_{i}$ are integers and $f^{-1}(p)=E=\sum_{i=1}^{n} E_{i}$, where each $E_{i}$ is an irreducible component.

Proof. First note that from Theorem 3.11 the exceptional divisor $E$ has a strictly pseudoconvex neighbourhood $V$. For a sufficiently small neighbourhood $U$ of $p$, the sheaf $N_{\mathcal{F}}^{\otimes k}$ is trivial. For $U$ and $V$ small enough, we can assume $f^{-1}(U)=V$.

Since $N_{\mathcal{G}}^{\otimes k}=f^{*} N_{\mathcal{F}}^{\otimes k}$ is trivial over $V-E$, then there is a holomorphic section without zeros $s$ of the bundle $N_{\mathcal{G}}^{\otimes k}$ over $V-E$. The Lemma 3.14 above imply that 
$s$ can be extended as a meromorphic section $\tilde{s}$ over all $V$, with poles or zeros along $E$. This finish the proof.

Proof of the Theorem 3.13. Let $f: Y \rightarrow X$ be a resolution singularity at $p \in X$ such that the induced foliation $\mathcal{G}=\pi^{*} \mathcal{F}$ is reduced along $E=f^{-1}(p)$. Suppose, by contradiction, that there is no singular point of $\mathcal{G}$ in $E-\operatorname{Sing}(E)$. Since $N_{\mathcal{F}}$ is a $\mathbb{Q}$-Gorenstein sheaf, it follows from Proposition 3.15 that, in neighbourhood of $E$,

$$
N_{\mathcal{G}}^{\otimes k}=\mathcal{O}_{Y}\left(\sum_{i=1}^{n} a_{i} E_{i}\right)
$$

where $k>0$, and the $a_{i}$ are integers, and $E=\sum_{i=1}^{n} E_{i}$, each $E_{i}$ being an irreducible component of $E$.

Since there is no saddle-node in $E$, then, by the intersection formula of the normal bundle given by Theorem 2.6, we have $N_{\mathcal{G}} \cdot E_{j}=E \cdot E_{j}$, that is, $\left(N_{\mathcal{G}}-E\right) \cdot E_{j}=$ 0 for $j=1, \ldots, n$. Then $\left(\sum_{i=1}^{n} a_{i} E_{i}-k E\right) \cdot E_{j}=0$, that is, $\left(\sum_{i=1}^{n}\left(a_{i}-k\right) E_{i}\right) \cdot E_{j}=0$ for $j=1, \ldots, n$. Hence

$$
\left(\sum_{i=1}^{n}\left(a_{i}-k\right) E_{i}\right)^{2}=0 .
$$

Therefore, since the matrix of intersection of $E$ is negative definite (Grauert's Criterion 3.4), $a_{i}=k$ for $i=1, \ldots, n$, that is,

$$
N_{\mathcal{G}}^{\otimes k}=\mathcal{O}_{Y}\left(k \sum_{i=1}^{n} E_{i}\right)=\mathcal{O}_{Y}(k E)
$$

Hence by Theorem 3.12 there is a singular point of $\mathcal{G}$ in $E-\operatorname{Sing}(E)$ and separatrix trough it not supported on $E$, which is projected over a separatrix of $\mathcal{F}$ trough $p$.

The proof just presented shows something more:

Theorem 3.16. Let $\mathcal{F}$ be a foliation on the singular normal surface $X$. Let $f: Y \rightarrow X$ be a resolution singularity at $p \in X$ such that the induced foliation $\mathcal{G}=f^{*} \mathcal{F}$ is reduced along $E=f^{-1}(p)$. If $\mathcal{G}$ has no saddle-node in the exceptional divisor $E$ and

$$
\left.N_{\mathcal{G}}\right|_{E} ^{\otimes k}=\left.\mathcal{O}_{Y}\left(\sum_{i=1}^{n} a_{i} E_{i}\right)\right|_{E}
$$

where $a_{1}, \ldots, a_{n} \in \mathbb{Z}$, then $\mathcal{F}$ has a separatrix trough $p$. 


\section{References}

1. Barth, W., Peters, C. and Van de Ven, A., Compact Complex Surfaces, Springer, 2003. MR0749574

2. Brunella, M., Birational Geometry of Foliations. Monografías de Matemática, Instituto de Matemática Pura e Aplicada (IMPA), Rio de Janeiro, 2000, 138 pp. MR1948251

3. Camacho, C., Quadratic forms and holomorphic foliations on singular surfaces, Math. Ann. 282 (1988), 177-184. MR0963011

4. Camacho, C. and SAD, P., Invariant varieties through singularities of holomorphic vector fields, Ann. of Math. 115 (1982), 579-595. MR0657239

5. CANO, J., Construction of invariant curves for singular holomorphic vector fields, Proc. Amer. Math. Soc. 125 (1997), 2649-2650. MR1389507

6. Grauert, H., Über modifikationen und exzeptionelle analytische Mengen, Math. Ann. 146 (1962), 331-368. MR0137127

7. Lins-Neto, A., Construction of singular holomorphic vector fields and foliations in dimension two, J. Differential Geom. 26 (1987), 1-31. MR0892029

8. Mattei, J.-F. and Moussu, R., Holonomie et intégrales premières, Ann. Sci., ENS 13 (1980), 469-523. MR0608290

9. Sebastiani, M., Sur l'existence de séparatrices locales des feuilletages des surfaces, An. Acad. Brasil. Ciênc. 69 (1997), 159-162. MR1754036

10. Toma, M., A short proof of a theorem of Camacho and Sad, Enseign. Math. 45 (1999), 311-316. MR1742334

11. Ueda, T., Neighborhood of a rational curve with a node, Publ. RIMS, Kyoto Univ. 27 (1991), 681-693. MR1140682

12. Voisin, C., Hodge Theory and Complex Algebraic Geometry, I, Cambridge Studies in Advanced Mathematics 76, Springer, 2002. MR1967689

Edileno de Almeida Santos

Instituto de Ciência, Engenharia e Tec-

nologia

Universidade Federal dos Vales do Jequit-

inhonha e Mucuri - UFVJM

Campus do Mucuri

Teófilo Otoni, MG

Brazil

edileno.santos@ufvjm.edu.br

Received November 7, 2018

in revised form September 10, 2019 